\title{
Coulomb charging of a quantum dot in the presence of adiabatically transmitted edge channels
}

\author{
B.W. Alphenaar, A.A.M. Staring and H. van Houten \\ Phllps Research Laboratortes, Eindhoven, The Netherlands
}

I.K. Marmorkos and C.W.J. Beenakker

Instutuut-Lorentz, Untverstly of Letden, Leiden, The Netherlands

C.T. Foxon ${ }^{1}$

Philips Research Laboratories, Redhill, UK

\begin{abstract}
We present measurements of the conductance of a quantum dot in the presence of 2,1 and 0 adiabatically transmitted edge channels In all three cases periodic conductance oscillations are observed as a function of gate voltage By using the oscillations observed in the absence of transmitted edge channels as an clection counter we demonstrate that the oscillations observed in the presence of transmitted edge channels are due to the influence of Coulomb charging Usıng a simple model calculation we show that the dctivation energy for tunneling vid an intermediate state in the confined edge channel oscillates periodically as a function of the Fermı energy, in qualitative agreement with the experiment
\end{abstract}

\section{Introduction}

Using current lithographic techniques it is possible to fabricate a zero-dimensional conducting island, or quantum dot, in the two-dimensional electron gas (2DEG) of a gated AIGaAs/ GaAs heterostructure. These dots have generated a great deal of interest. At low temperatures, the influence of both the confined energy level spacing and the Coulomb charging energy can be observed in the dot conductance. A typical quantum dot device is shown in fig. 1(a). The dot is connected to two leads by adjustable quantum point contacts (QPCs), while the density is varied by the voltage $V_{\mathrm{B}}$ on an independent gate electrode. In a high magnetic field, the

Correspondence to $\mathrm{BW}$ Alphendar, Hitachı Cambridge Laboratory, Cambridge CB3 0HE, UK

${ }^{1}$ Present address Department of Physics, University of Nottıngham, Nottungham NG7 2RD, UK conductance of the QPCs is approximately given by [1]

$G_{\mathrm{pc}} \approx \frac{e^{2}}{h}\left(N_{\mathrm{trans}}+t\right)$,

with $N_{\text {trans }}$ the number of edge channels that are fully transmitted over the barrier in the constriction, and $t \leqslant 1$ the tunneling transmission probability of the $\left(N_{\text {trans }}+1\right)$ th edge channel. The $\left(N_{\text {tidns }}+1\right)$ th edge channel corresponds to the lowest index Landau level (LL) that is confined to the dot. Edge channels corresponding to higher index LLs are nearly completely reflected.

The states confined to the dot form a discrete energy spectrum, with average energy spacing $\delta E$. For a non-interacting electron gas, a peak in the conductance due to resonant tunneling is observed if an electron state in the dot lines up with the Fermi level in the leads, $E_{\mathrm{F}}$. This results in periodic conductance oscillations as $V_{\mathrm{B}}$ is varied and the energy spectrum moves through 

(a)

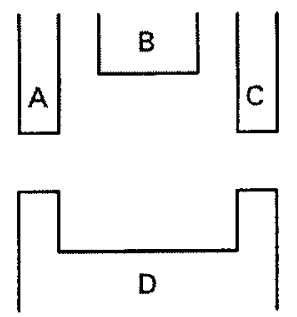

(b)

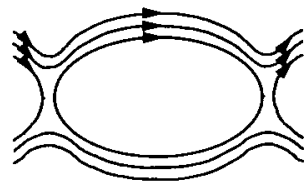

(c)

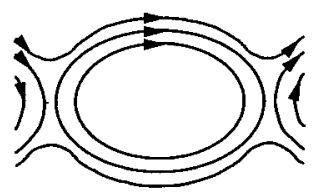

(d)

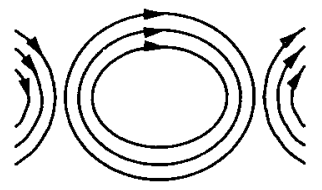

Fig 1 (a) Schematic drawing of the quantum dot devices The dimunsions of the rectangle formed by the gates is $750 \mathrm{~nm} \times 800 \mathrm{~nm}$ for dot I and $700 \mathrm{~nm} \times 900 \mathrm{~nm}$ for dot II (b) (d) Cuticnt paths through the dot in the picsence of 21 or 0 adiabatically tiansmitted edge channels as adjusted by $V_{B}$ and $V_{1}$

$E_{1}$ It has recently been demonstrated [2], however, that tunneling through a quantum dot can also be governed by the Coulomb charging energy for a single tunneling electron This effect is dominant if the capacitance of the $\operatorname{dot} C$ is small so that the charging energy $e^{3} / 2 C$ is substantially larger than $\delta E$ Since the charging eneigy varies periodically with the electron density in the dot, Coulomb interactions also produce conductance oscillations periodic in $V_{\mathrm{B}}$ (Coulomb blockade oscillations)

In a high magnetic field, single electron chaig ing effects are known to be important if the conductance of the point contacts $G_{p c}<e^{2} / h$ In this case edch edge channel is at least partidly backscattered and all transport through the dot is due to tunneling (fig $1(\mathrm{~d})$ ) If $G_{\mathrm{pc}}>e^{\mathrm{e}} / \mathrm{h}$, one or more edge channels are adiabatically transmitted through the dot ( 1 e $N_{11 \mathrm{~m}}=2$ or 1 as shown in higs 1(b) and (c)) Since these edge channels are extended rather than localized in the dot one might think that Coulomb charging is sup pressed Conductance oscillations observed in this regime as a function of magnetic field [3] and as a function of gate voltage [4] have thus been attributed to resonant tunneling, (or equivalently the Aharonov-Bohm effect [5])

In a recent paper [6], however, we demonstrated the importance of Coulomb charging in the presence of extended edge channels Here, we present more extensive datd from measurements of two additional quantum dot devices that confirm this conclusion In both devices, we observe periodic conductance oscillations as a function of gate voltage in the presence of 2,1 and 0 adiabatically transmitted edge channels Following the method of ief [6] we use the oscillations obseived in the full Coulomb blockade regime as an electron counter, and show that Coulomb charging has a strong influence on the period of the conductance oscillations, even in the presence of extended edge channels In addition, we present a theoretical calculation [7] of the activation energy for charge transfer from an extended to a localized channel, assuming an electron-electron interaction mediated by a Thomas-Fermi potential This is compared with the activation energy determined experimentally from the temperature dependence of the conductance minımd

\section{Experiment}

The geometry of our two quantum dot devices (dot I and dot II) is shown schematically in fig 1(d) [8] Four gates (labelled A-D) define a rectangle on the surface of an AlGaAs/GaAs heterostructure with a $2 \mathrm{DEG}$ of mobility $\mu \approx$ $10^{6} \mathrm{~cm}^{3} / \mathrm{Vs}$ and density $n_{s} \approx 3 \times 10^{11} \mathrm{~cm}^{2}$ The lithographic dimensions of dot I are $750 \times$ $800 \mathrm{~nm}^{\text {? }}$ and those of dot II are $700 \times 900 \mathrm{~nm}^{2}$ When the gates are negatively biased, a quantum dot is formed in the underlying $2 \mathrm{DEG}$, and is connected through two QPCs to two-dimensional 
leads. Two-terminal conductance measurements are made across the dot using an ac lock-in technique with an excitation voltage below $10 \mu \mathrm{V}$. In the experiments, the voltage on gate $\mathrm{D}$ is left fixed, and the voltages on gates $\mathrm{A}$ and $\mathrm{C}$ are adjusted to control the transmission through the tunnel barriers in the QPCs. The conductance is then measured as a function of the voltage on gate $\mathrm{B}$ which determines the electron density in the dot.

Fig. 2 shows the results of conductance measurements of dot $\mathrm{I}$ as a function of $V_{\mathrm{B}}$ for a magnetic field of $B=3.50 \mathrm{~T}$ and a temperature of $50 \mathrm{mK}$. The QPCs are adjusted so that there are $N_{\text {trans }}=2,1$ and 0 edge channels adiabatically transmitted through the dot for traces (a), (b) and (c), respectively. This corresponds to the following QPC conductances: (a) $2 e^{2} / h<G_{\mathrm{pc}}<$ $3 e^{2} / h$, (b) $e^{2} / h<G_{\mathrm{pc}}<2 e^{2} / h$, and (c) $G_{\mathrm{pc}}<$ $e^{2} / h$. Each trace shows a series of nearly periodic oscillations in the conductance as a function of $V_{\mathrm{B}}$, however the period of the oscillations varies from trace to trace. There are 53 oscillations in (a), 80 in (b), and 102 in (c), indicating that the period in (a) is about twice that in (c), while the period in (b) is about 1.2 times that in (c).

Fig. 3 compares the conductance oscillations from measurements made at four different magnetic fields $(2.75,3.50,4.20$ and $4.60 \mathrm{~T})$. In each case the QPCs are adjusted so that $2 e^{2} / h<$ $G_{\mathrm{pc}}<3 e^{2} / h$, i.e. $N_{\text {trans }}=2$. Between 2.75 and $4.60 \mathrm{~T}$ periodic conductance oscillations are observed whose period increases with magnetic field; the amplitude of the oscillations drops off rapidly outside of this field range. In the $N_{\text {trans }}=$ 1 case $\left(e^{2} / h<G_{\mathrm{pc}}<2 e^{2} / h\right)$ (not shown) oscillations are observed for magnetic fields less than $7 \mathrm{~T}$ and the period of the oscillations again increases with increasing magnetic field. For the $N_{\text {trans }}=0$ case, however, oscillations are observed irrespective of the magnetic field and the period of the oscillations is field independent.

\section{Model}

The Coulomb charging energy for the $\operatorname{dot} e^{2} /$ $2 C \simeq e^{2} / 8 \epsilon d \approx 0.25 \mathrm{meV}$, is greater than $k T$ for temperatures up to $3 \mathrm{~K}$. Thus the very regular oscillations observed in the absence of adiabatically transmitted edge channels (the bottom trace in fig. 2) can safely be attributed to the Coulomb blockade effect. The gate voltage separation between peaks is $\approx e / C_{\mathrm{gate}}$ where $C_{\mathrm{gatc}}$, the capacitance between gate $B$ and the dot, is assumed to be independent of $G_{\mathrm{pc}}$. This is reasonable since $G_{\mathrm{pc}}$ is much more sensitive to changes in voltages $V_{\mathrm{A}}$ and $V_{\mathrm{C}}$ than are the size of the dot and the dot-gate separation (which together determine $C_{\mathrm{gatc}}$ ). Since each Coulomb blockade peak corresponds to the removal of one electron from the dot, this allows us to use the Coulomb blockade oscillations in a novel way: as a tool to determine the mechanism that governs the period of the oscillations seen at higher barrier transparencies. The number of electrons per peak for the upper traces in fig. 2 can be determined by dividing the number of Coulomb blockade peaks counted for $N_{\text {trans }}=0$ into the number of peaks for $N_{\text {trans }}=1$ or 2 . The results of this procedure performed on a set of measurements of the type shown in figs. 2 and 3 are plotted versus magnetic field in fig. 4. The

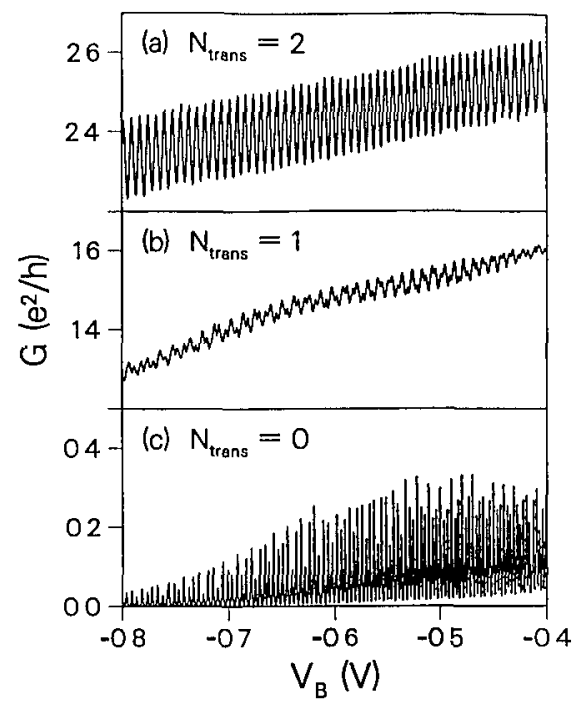

Fig 2 Conductance of dot I as a function of the voltage on gate $\mathrm{B}$ for $B=35 \mathrm{~T}$ and $T=50 \mathrm{mK}$ In the top, middle, and bottom traces (a)-(c), QPCs are adjusted to conductances of 25,15 and $05 e^{2} / h$, respectively The three cases correspond to the three possible current paths shown in figs $1(\mathrm{~b})-(\mathrm{d})$ 


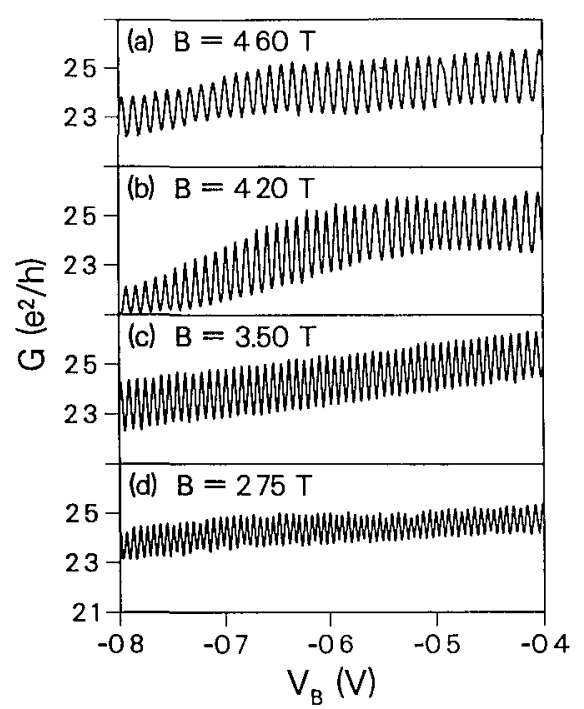

Fig 3 Conductance of dot $I$ as a function of the voltage on gate $\mathrm{B}$ for $G_{\mathrm{p}}=25 e^{\lambda} / h\left(N_{\mathrm{tum}}=2\right)$ at four different magnetıc fields $(T=50 \mathrm{mK})$

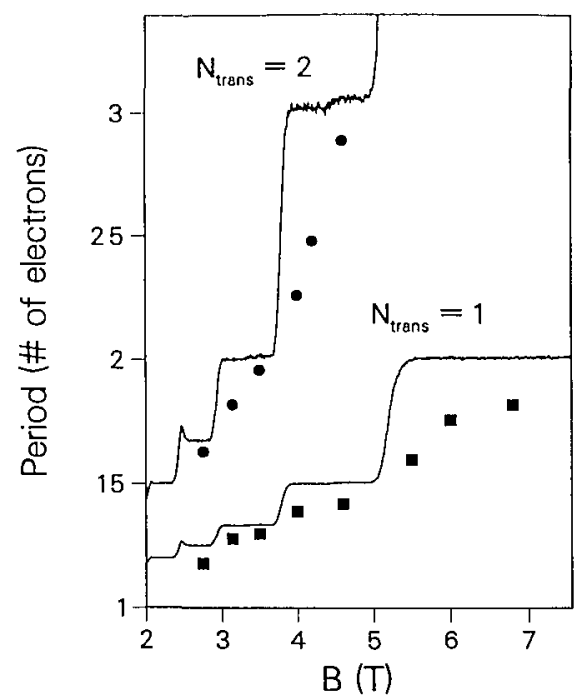

Fig 4 Period of the conductance oscillations (in units of electrons per peak) versus magnetic field for $G_{\mathrm{pu}}=25 e^{2} / \mathrm{h}$ (circles) and $G_{\mathrm{m}}=15 e^{2} / h$ (squares) The solid lines are theoretical fits for $N_{\text {trin }}=2,1$ adiabatically transmitted edge channels (see text)

number of electrons per peak is considerably larger for $N_{\mathrm{trams}}=2$ than for $N_{\mathrm{tran}}=1$. In both cases, an increase in magnetic field results in an increase in the number of electrons per peak.

We now consider the periodic conductance oscillations for $G_{\mathrm{pc}}>e^{2} / h$ (traces (a) and (b) in fig. 2). The simplest possibility is to ignore Coulomb charging. As discussed in the introduction, then a peak in the conductance is observed due to resonant tunneling when an electron state of the outer most confined LL lines up with the Fermi energy in the leads. The frequency at which this occurs corresponds to the rate at which electrons are removed from the outermost confined LL. The total number of spin-split LLs in the dot $N_{\text {dot }}$ is made up of the $N_{\text {cont }}$ LLs of guiding center energy $E_{\mathrm{g}}$ below the barrier height $E_{\mathrm{b}}$ and the $N_{\text {trans }}$ additional LLs that are occupied in the dot but fully transmitted over the barriers in the QPCs $\left(E_{\mathrm{g}}<E_{\mathrm{b}}\right)$. Assuming that both the $N_{\text {cont }}$ and the $N_{\text {trans }}$ LLs are depleted at about the same rate, the number of electrons per peak should be simply $N_{\text {dot }}$. This argument predicts that the number of electrons per peak should decrease, rather than increase with magnetic field, in contradiction with the observed results (fig. 4). This discrepancy could in principle be eliminated if resonant tunneling through electron states of the confined LLs with index higher than $N_{\text {tran }},+1$ also contributes to the conductance of the dot. The tunneling rate, however, decreases exponentially with decreasing $E_{g}[9]$, thus, there should be an order of magnitude modulation of conductance peak heights due to resonant tunneling through states belonging to consecutive LLs confined in the dot [10]. This is not observed in our experiment, however [11].

We can model the results of fig. 4, though, if we take Coulomb charging into account for $G_{\mathrm{pc}}>e^{2} / h$. We extend recent arguments for the $N_{\text {trans }}=0$ case [12] to our problem by considering a separate Coulomb charging energy of the $N_{\text {conf }}$ LLs existing in the presence of the adiabatically transmitted edge channels. This is reasonable, since a magnetically induced tunnel barrier consisting of an incompressible electron gas region exists between each of the edge channels. Resonant tunneling electrons thus face a non-zero Coulomb charging energy associated with a change in the electron population of the confined LLs. This leads to Coulomb blockade oscillations as a function of gate voltage with a period 
corresponding to the removal of an electron from any one of the $N_{\text {conf }}$ LLs. The removal of electrons from one of the $N_{\text {trans }}$ LLs in the dot does not give rise to a conductance peak because charge in these levels is not localized and can therefore be changed continuously. This implies that

$\frac{\text { electrons }}{\text { peak }}=\frac{N_{\text {conf }}+N_{\text {trans }}}{N_{\text {cont }}}=\frac{N_{\text {dot }}}{N_{\text {conf }}}$.

Fig. 4 shows solutions of eq. (2) for $N_{\text {trans }}=1$ and $N_{\text {trans }}=2$. We determine $N_{\text {dot }}$ by measuring the conductance of the dot with the two QPCs completely open $\left(V_{\mathrm{A}}=V_{\mathrm{C}}=0\right)$. The agreement between our model and the experimental results is very good. In both cases, the number of electrons per peak is seen to increase as $N_{\text {dot }}$ decreases from 5 to 3 . This comparison demonstrates then that Coulomb charging effects dominate even though $G_{\mathrm{pc}}>e^{2} / h$.

\section{Activation energy calculation}

In this section we present a simple model calculation of the activation energy for single electron tunneling in the presence of adiabatically transmitted edge channels [7]. We begin by considering a dot containing two edge channels, one confined and one extended (inset (a) of fig. 5). We seek the activation energy for an electron tunneling from the leads into the confined edge channel (indicated by the arrow in the inset (a) of fig. 5) as a function of the Fermi energy in the leads. Since the extended edge channel is connected directly to the leads, adding an electron to the confined edge channel results in the appearance of a positive image charge on the extended edge channel. The change in the charge distribution of the dot is thus equivalent to a single electron tunneling from the extended edge channel into the confined edge channel, as shown in inset (b). What is neglected here is a possible contribution from image charges in the leads. In other words, we consider only the self-capacitance of the dot, and neglect the

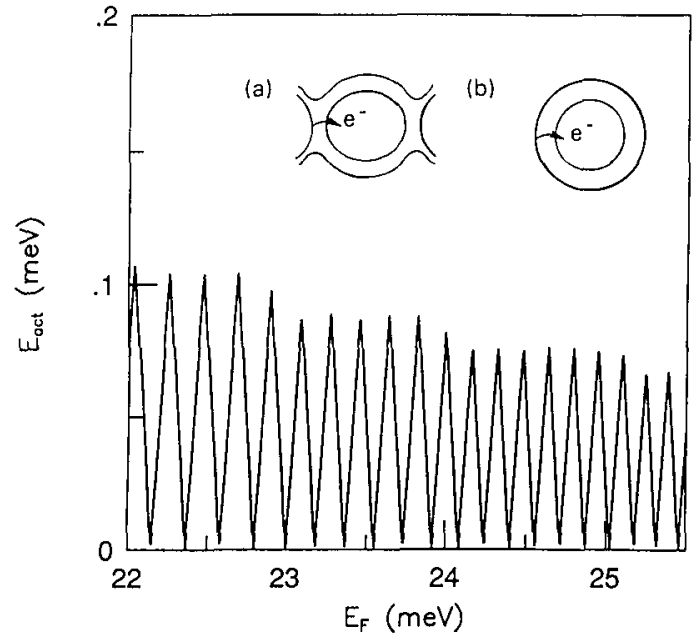

Fig 5 Activation energy as a function of Fermı energy for a quantum dot with two edge channels occupied, one extended and one localized The inset is discussed in the text

capacitance to the leads, which originates at the tunnel barriers.

For the total electrostatic energy of the dot we use the self-consistent Thomas-Fermi model proposed by McEuen et al. [12],

$$
\begin{aligned}
U= & \sum_{n, s}\left(\omega_{\mathrm{c}}\left(n+\frac{1}{2}\right)+g \mu_{\mathrm{B}} B s\right) N_{n s}+\int \mathrm{d}^{2} \boldsymbol{r} V_{\mathrm{ext}}(\boldsymbol{r}) \rho(\boldsymbol{r}) \\
& +\frac{1}{2} \int \mathrm{d}^{2} \boldsymbol{r} \int \mathrm{d}^{2} \boldsymbol{r}^{\prime} V_{\mathrm{ee}}\left(\boldsymbol{r}-\boldsymbol{r}^{\prime}\right) \rho(\boldsymbol{r}) \rho\left(\boldsymbol{r}^{\prime}\right) .
\end{aligned}
$$

The index $n=0,1,2, \ldots$ labels the Landau levels, the index $s= \pm \frac{1}{2}$ labels the spins polarization. The sum over $n$ and $s$ gives the kinetic and Zeeman energy of the $N_{n s}$ electrons in each Landau level $\left(\omega_{\mathrm{c}}=\mathrm{eB} / \mathrm{m}\right.$ is the cyclotron frequency and $g \mu_{\mathrm{B}} B$ the Zeeman splitting). The integrals over the areal electron density $\rho(\boldsymbol{r})$ give the confinement and interaction energy in the approximation of a slowly varying electron density. We take a parabolic confining potential $V_{\text {ext }}(r)=\frac{1}{2} m \omega_{0}^{2} r^{2}$. As in ref. [12], the electronelectron interaction potential is modeled by

$V_{\mathrm{ee}}(r)=\frac{e^{2}}{\epsilon\left(r^{2}+\delta^{2}\right)^{1 / 2}}-\frac{e^{2}}{\epsilon\left(r^{2}+4 d^{2}\right)^{1 / 2}}$,

to include the effects of the finite thickness $\delta$ of 
the $2 \mathrm{DEG}$ layer and the image charge on a gate clectrode at a distance $d$ above the 2DEG. In our numcrical work we took $\hbar \omega_{0}=0.9 \mathrm{meV}, \delta=$ $50 \AA, d=100 \AA$ and dielectric constant $\epsilon=13.6$.

Our qualitative conclusions are not sensitive to the specific value of the parameters used in our numerical work. However we had difficulty doing a quantitative comparison with the experiment. In the expcriment the distance between the gate electrode and the $2 \mathrm{DEG}$ is around $1000 \AA$. We found that using $d=1000 \AA$ in our model calculation requires a much too large Fermi energy in the reservoirs, in order to populate the two lowest Landau levels in the dot. To compromise, we have artificially reduced $d$ to $100 \AA$, but still our $E_{\mathrm{F}}$ is a factor of two larger than in the experiment. Furthermore, we limit ourselves to not more than two populated Landau levels. We believe that the reason that our model overestimates the electrostatic energy is our neglect of the capacitance to the leads.

To determine the ground state of the quantum dot in equilibrium with electron reservoirs at Fermi energy $E_{\mathrm{F}}$, we minimize the thermodynamic potential

$\Omega=U-N E_{\mathrm{F}}$,

where $N=\int \mathrm{d}^{2} r \rho(r)$ is the number of electrons in the dot. The number of electrons with quantum numbers $n, s$ is given by

$N_{\mu, s}=\int \mathrm{d}^{2} r \rho_{m}(\boldsymbol{r})$,

with $N=\Sigma_{n s} N_{n s}$. The number $N_{n}$ is constrained to be an integer for a confined edge channel, whereas it is an unconstrained positive real number for an extended edge channel. The Landau level degeneracy constrains the particle density $\rho_{t s}(r)$ of electrons with quantum numbers $n, s$ to the interval

$0 \leqslant \rho_{n, s}(\boldsymbol{r}) \leqslant \frac{e B}{h}$.

The minimization of $\Omega$ subject to the above constraints is carried out numerically, and yiclds a ground state thermodynamic potential $\Omega_{g}$, with the corresponding density distributions per
Landau level. To obtain the activation energy $E_{\mathrm{act}}$ we repeat the minimization twice, subject to the additional constraint that the total number of electrons in the localized edge channels is either one more or one less than the number $N_{0}$ in the ground state configuration. This yields two additional thermodynamic potentials, $\Omega_{+}$and $\Omega_{-}$. The activation energy is defined by $E_{\mathrm{act}}=$ $\min \left(\Omega_{+}-\Omega_{\mathrm{g}}, \Omega_{-}-\Omega_{\mathrm{g}}\right)$, and is non-negative by construction. If $E_{\mathrm{act}}=0$, either the process $N_{0} \rightarrow N_{0}+1 \rightarrow N_{0} \rightarrow \ldots$ or the process $N_{0} \rightarrow$ $N_{0}-1 \rightarrow N_{0} \rightarrow \ldots$ costs zero energy, that is to say, an electron can tunnel through the dot via an intermediate state in the confined edge channels without cost of energy.

\section{Discussion}

In fig. 5 we plot the model calculation of $E_{\text {act }}$ as a function of $E_{\mathrm{F}}$ for a quantum dot with the $n=0$ edge channel extended and the $n=1$ edge channel confined at $B=3.5 \mathrm{~T}$. Triangularly shaped oscillations are observed in $E_{\text {act }}$ that decrease in magnitude with increasing $E_{\mathrm{F}}$. In order to test the validity of our calculation, we compare it with the temperature dependence of

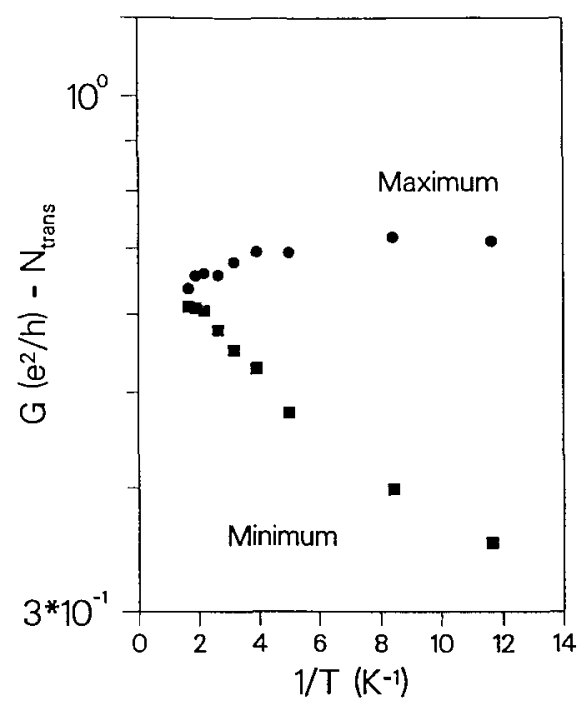

Fig. 6. Tempcrature dependence of a maximum and a minimum in the conductance of dot II, as a function of inverse temperature for $B=3.5 \mathrm{~T}$ and $N_{\mathrm{tram}}=2$. 

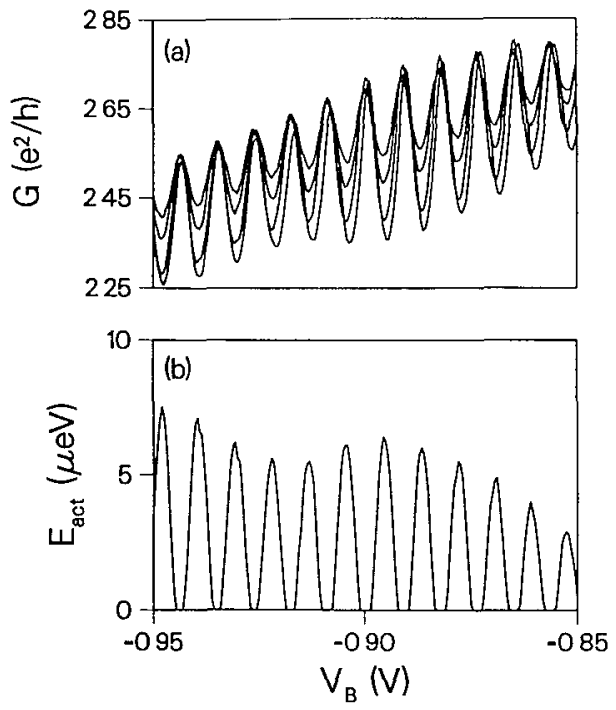

Fig 7 (a) Conductance of dot II as a function of the voltage on gate B at 380310250 and $200 \mathrm{mK}$ (top to bottom) Measurements are made at $B=35 \mathrm{~T}$ and $N_{\mathrm{tr} n}=2$ (b) Activation energy calculated from the data in (a)

the conductance oscillations of $\operatorname{dot}$ II for $B=$ $35 \mathrm{~T}, N_{\text {trans }}=2$ and $N_{\text {dot }}=4$ The experimental results are summarized in figs 6 and 7 Fig 6 shows the temperature dependence of a conductance maximum and minımum obtained from the conductance measurements shown in fig 7(a) The conductance minima show an activated temperature dependence while the maxima are virtually temperature independent In fig 7(b) we plot the activation energy as a function of $V_{\mathrm{B}}$ as calculated from the results of fig 7(a) Although qualitatively the experiment and theory agree, the peak activation energy in fig $7(\mathrm{~b})$ is an order of magnitude smaller than that calculated theoretically (fig 5)

It appears that the model is unable to make a quantitative prediction of the activation energy Most likely, the main reason for the discrepancy is our neglect of the dot-lead capacitance of the tunnel barriers relative to the self-capacitance of the quantum dot Recent experimental [13] and theoretical [14] work, however, suggest that this capacitance can be substantial even for small barrier conductances

In conclusion, we have studied the conductance of a quantum dot in the presence of 2,1 or
0 adiabatically transmitted edge channels We observe periodic conductance oscillatıons as a function of gate voltage in all three cases, and demonstrate that the number of electrons added to the dot per peak is determined by the ratio of the total number of LLs in the dot to the number of LLs confined to the dot Our results demonstrate that the formation of LLs in a high magnetic field causes single electron charging effects to be of importance for barrier conductances greater than $e^{2} / h$ Usıng a simple model calculation we have shown that the activation energy for tunneling via an intermediate state in a confined edge channel oscillates periodically as a function of $E_{\mathrm{F}}$ A more detarled calculation is needed in order to make quantitative compar1sons with the experimentally determined charging energy

\section{Acknowledgements}

The authors would lıke to thank P L McEuen and LW Molenkamp for useful discussions, and M A A Mabesoone and O J A Buyk for expert technical assistance Research at Leiden University is supported by the Dutch Science Foundation NWO/FOM

\section{References}

[1] B J van Wees, L P Kouwenhoven $H$ van Houten, CW J Beenakker J E Moolj C T Foxon and J J Harris Phys Rev B 38 (1988) 3625

[2] For a recent review of Coulomb blockade in semı conductor nanostructures see $\mathrm{H}$ van Houten $\mathrm{CWJ}$ Beenakker and A A M Staring in Single Charge Tunnelıng, eds $\mathrm{H}$ Grabert and $\mathrm{MH}$ Devoret, NATO ASI Series B 294 (Plenum, New York 1992)

[3] B J van Wees L P Kouwenhoven C J PM Harmans J G Willamsons, C E Timmering M E I Broekdart C T Foxon and J J Harris, Phys Rev Lett 62 (1989) 2523

[4] R J Brown C G Smith, M Pepper, M J Kelly R Newbury, $\mathrm{H}$ Ahmed, D G Haskos, J E F Frost, D C Peacock, D A Ritchie and G A C Jones J Phys C 1 (1989) 6291

[5] J K Jain, Phys Rev Lett 60 (1988) 2074, U Sivan and Y Imry Phys Rev Lett 61 (1988) 1001

[6] B W Alphenaar A A S Staring, H van Houten 
M A A Mabesoone, O J A Buyk and C T Foxon, Phys Rev B 46 (1992) 7236

[7] I K Marmorkos and CWJ Beenakker, Phys Rev B 46 (1992) 15562

[8] For an exact description of the geometry of dot I and dot II sec A A M Starıng, B W Alphenaar, H van Houten, LW Molenkamp, O J A Buyk, M A A Mabesoone and C T Foxon, Phys Rev B 46 (1992) 12869 ,

A A M Staring, J G Willamson, $H$ van Houten, C W J Beenakker, L P Kouwenhoven and C T Foxon, Physica B 175 (1991) 226

[9] H A Fertıg and B I Halperm, Phys Rev B 36 (1987) 7969

[10] Y Meir, N S Wingreen and P A Lee, Phys Rev Lett 66 (1991) 3048

CW J Beenakker, Phys Rev B 44 (1991) 1646

[11] The more modest amplitude modulation of the conductance peaks that we observe in the middle and bottom traces of fig 2 can be explained in terms of tunneling through the confined states associated with only the $\left(N_{\text {trins }}+1\right)$ th LL The fact that a more dramatic amplitude modulation is not observed suggests that we are in the near classical regime where $k T \geqslant \delta E$, the average confined level spacing in the dot see A A M Staring, B W Alphenadr, $H$ van Houten, LW Molenkamp, O J A Buyk, M A A Mabesoone and C T Foxon, Phys Rev B 46 (1992) 12869

[12] P L McEuen, E B Foxman, J Kinaret, U Merrav, M A Kastner, N S Wingreen and S J Wind, Phys Rev B 45 (1992) 11419

[13] E B Foxman, P L McEuen, U Melrav, Ned S Wingreen, Yigal Meır, Paul A Belk, N R Belk, M A Kastner and S J Wind, Phys Rev B, to be published

[14] Ned S Wingreen and Yigal Merr, NATO Advanced Research Workshop on Physics of Few-Electron Nanostructures, The Netherlands, 1992 